\title{
Granulomatous keratic precipitates in birdshot retinochoroiditis
}

\author{
Pascal B. Knecht • Marina Papadia • \\ Carl P. Herbort
}

Received: 26 September 2012/ Accepted: 3 October 2012/Published online: 19 October 2012

(C) Springer Science+Business Media Dordrecht 2012

\begin{abstract}
With the purpose of facilitating clinical studies of this infrequent disease, an expert panel published research criteria for birdshot retinochoroiditis (RCBRC). The aim of our study was to investigate the sensitivity of the exclusion criteria of the RCBRC as applied to all patients seen in our center with a diagnosis of BRC. This was a single center retrospective study involving all patients with an ocular inflammatory disease seen at the Centre for Ophthalmic Specialized Care, Lausanne, Switzerland, between 1995 and 2012. The percentage of patients with a diagnosis of BRC was identified. The exclusion criteria of the RCBRC were applied to all patients and the percentage of patients with absence of keratic
\end{abstract}

P. B. Knecht · C. P. Herbort ( $\square)$

Retinal and Inflammatory Eye Diseases,

Centre for Ophthalmic Specialized Care (COS),

Rue de la Grotte 6, 1003 Lausanne, Switzerland

e-mail: carl.herb@bluewin.ch

P. B. Knecht

e-mail: pascalknecht@gmx.ch

P. B. Knecht

Department of Ophthalmology, University Hospital

Zurich, Zurich, Switzerland

M. Papadia

Eye Clinic, Department of Neurosciences, Ophthalmology

and Genetics, University of Genova, Genova, Italy

C. P. Herbort

University of Lausanne, Lausanne, Switzerland precipitates (KPs) and absence of posterior synechiae were calculated. Out of 1,504 new patients, 25 patients (1.66\%; 19 female, 6 male) were diagnosed with BRC and 19 patients had sufficient data to be included in the study (1.26\%, 13 female). All patients were positive for HLA-A29 testing. The sensitivity of the RCBRC with respect to the exclusion criteria applied to our patient cohort was $84.2 \%$ due to the exclusion of three patients showing KPs. Our study supports the motion to re-evaluate the RCBRC, since granulomatous KPs can be present and are more frequent than previously estimated and stringent application of the criteria would lead to a substantial loss of study patients.

Keywords Birdshot retinochoroiditis .

Granulomatous uveitis · Keratic precipitates ·

Posterior synechiae

\section{Introduction}

Birdshot retinochoroiditis (BRC), described in 1980 by Ryan and Maumenee [1], is a bilateral retinochoroidal inflammatory disease without any known systemic involvement. It is a rare disease affecting mainly Caucasian patients. It has been suggested that this predilection is due to a higher prevalence of HLAA29. The proportion of BRC patients in European and North American uveitis series is $0.6-1.5 \%$ of uveitis cases [2-6]. Typically, BRC affects age groups from 
30-70 years with a median age around 50 years and with a strong gender predilection for women with a F/M ratio of 3/2 [7].

Histopathology has revealed that the choroidal stroma is one site of inflammation, while the choriocapillaris and the pigment epithelium are intact [8]. The second independent site of inflammation is the retina, showing a massive vasculitis at the onset of the disease [8]. The latter histopathological investigation as well as a recent report did not identify granulomatous histological features [9].

With the purpose of facilitating clinical studies of this infrequent disease, an expert panel published research criteria for BRC (RCBRC) as a consensus statement in 2006 [10]. Beside the mandatory bilaterality, typical signs for BRC like the presence of at least three peripapillary 'birdshot lesions' inferior or nasal to the optic disk in one eye need to be met. Furthermore, they proposed two exclusion criteria, both related to anterior chamber inflammation-keratic precipitates (KPs) and posterior synechiae (PS).

The aim of our retrospective study was to investigate the sensitivity of the exclusion criteria of the RCBRC as applied to all patients seen at our center with a diagnosis of BRC.

\section{Methods}

This was a single center retrospective study involving all patients with an ocular inflammatory disease seen at the Centre for Ophthalmic Specialized Care, Lausanne, Switzerland, between 1995 and 2012. All patients underwent complete routine work-up applied to patients with posterior uveitis. The study was performed in accordance with the Declaration of Helsinki.

The percentage of patients with a diagnosis of BRC was identified. Our diagnostic criteria for BRC included vitreitis and retinal vasculitis in either or both eyes, visual field anomalies in either or both eyes, stromal choroiditis as evidenced by indocyanine green angiography (ICGA) in both eyes and the presence of HLA-A29. An additional, non-obligatory diagnostic criterion indicating more advanced disease was the presence of rice-shaped depigmented 'birdshot lesions'. The exclusion criteria of the RCBRC were applied to all patients and the percentage of patients with absence of KPs and absence of PS were calculated.
Statistical analysis was performed using the statistics software GraphPad Prism (Version 5.02, GraphPad Software Inc., San Diego, C, USA).

\section{Results}

Out of 1,504 new patients with ocular inflammatory conditions seen between 1995 and 2012, 25 patients (1.66\%; 19 female, 6 male) were diagnosed with BRC and 19 patients had sufficient data to be included in the study $(1.26 \%$, 13 female). All patients were Caucasian. Mean age at onset was $41 \pm 4.4$ years with a mean follow-up of $64 \pm 38.6$ months.

All patients were positive for HLA-A29 testing.

Three out of 19 patients would not be allowed to be included in a BRC study (15.8\%), although they had all typical features of BRC. The sensitivity of the RCBRC with respect to their exclusion criterion as

Table 1 Application of the research criteria for birdshot retinochoroidits

\begin{tabular}{llll}
\hline Patient No. & KP & PS & $\begin{array}{l}\text { HLA-A29- } \\
\text { positive }\end{array}$ \\
\hline 1 & No & No & Yes \\
2 & No & No & Yes \\
3 & No & No & Yes \\
4 & No & No & Yes \\
5 & No & No & Yes \\
6 & No & No & Yes \\
7 & No & No & Yes \\
8 & No & No & Yes \\
9 & No & No & Yes \\
10 & No & No & Yes \\
11 & No & No & Yes \\
12 & Yes & No & Yes \\
13 & No & No & Yes \\
14 & No & No & Yes \\
15 & No & No & Yes \\
16 & No & No & Yes \\
17 & Yes & No & Yes \\
18 & Yes & No & Yes \\
19 & No & No & Yes \\
Percentage of & $84.21 \%$ & $100 \%$ & $100 \%$ \\
patients meeting & & & \\
the criteria & & & \\
\hline$K P$ & & $P S$ & \\
\hline & & & fos
\end{tabular}

$K P$ keratic precipitate formation, $P S$ posterior synechiae formation 
applied to our patient cohort was $84.2 \%$ due to the exclusion of three patients showing KPs (Table 1). On the other hand, none of our patients developed PS, representing a highly sensitive criterion in our cohort (Table 1).

\section{Case reports}

Patient 12 presented at the age of 61 years, with onset 7 years earlier. She showed trace anterior cells on both sides. Two KPs were seen in the left eye which also showed 2 + vitreous infiltration. These KPs were seen while the patient was not yet under treatment. Laser flare photometry measurements showed $12.7 \mathrm{ph} / \mathrm{ms}$ in the right eye and $9.6 \mathrm{ph} / \mathrm{ms}$ in the left eye. On fundus examination characteristic bilateral oval depigmented 'birdshot' lesions could be seen (Fig. 1) associated with bilateral retinal vasculitis on fluorescein angiography (FA) and bilateral typical ICGA signs for stromal choroiditis including hypofluorescent dark dots (HDDs), fuzziness of stromal choroidal vessels and late diffuse choroidal hyperfluorescence in an HLA-A29-positive patient. The KPs disappeared once the patient was administered dual inflammation suppressive therapy (IST) consisting of systemic steroids and cyclosporine.

Patient 17 presented at the age of 54 years, 3 years after onset of symptoms. Rare cells could be seen in the anterior chamber with flare values of $12.9 \mathrm{ph} / \mathrm{ms}$ in the right eye and $14.7 \mathrm{ph} / \mathrm{ms}$ in the left eye. One single

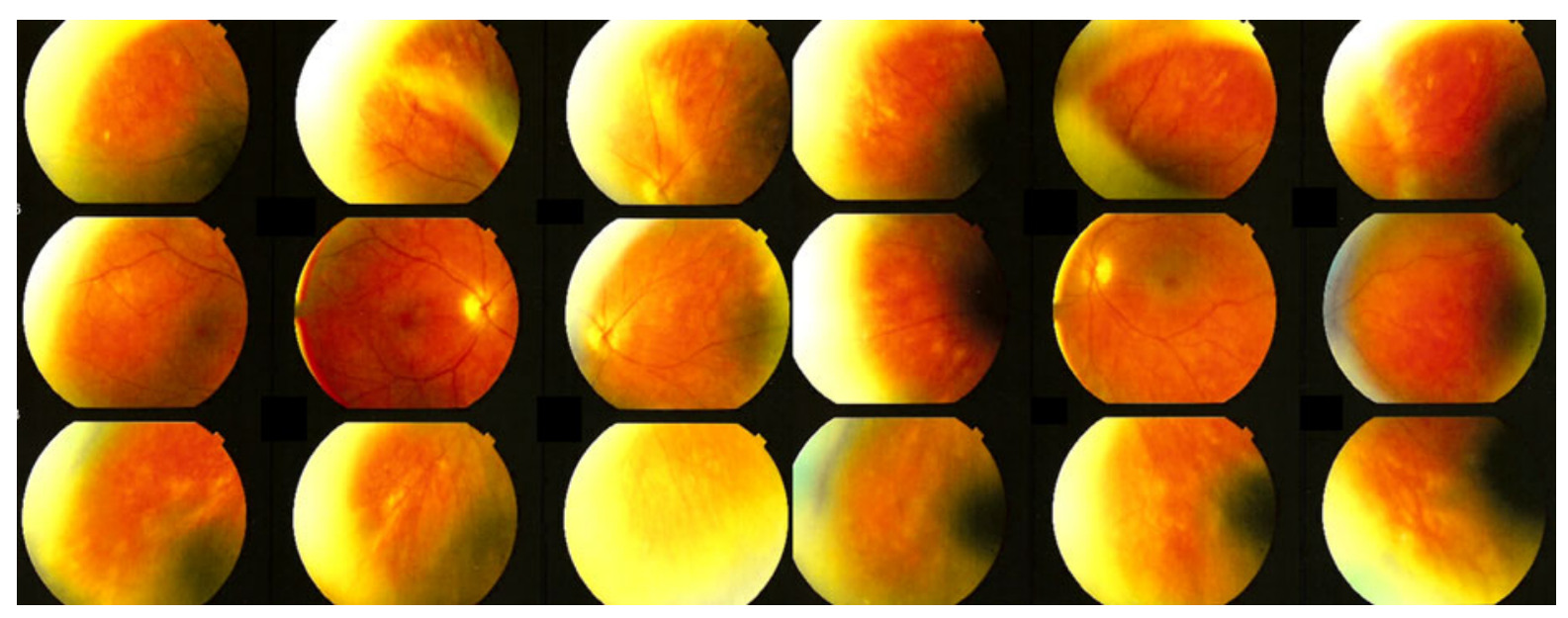

Fig. 1 Fundus photographs of patients 12 showing typical oval-shaped depigmented fundus lesions distributed in the mid-periphery

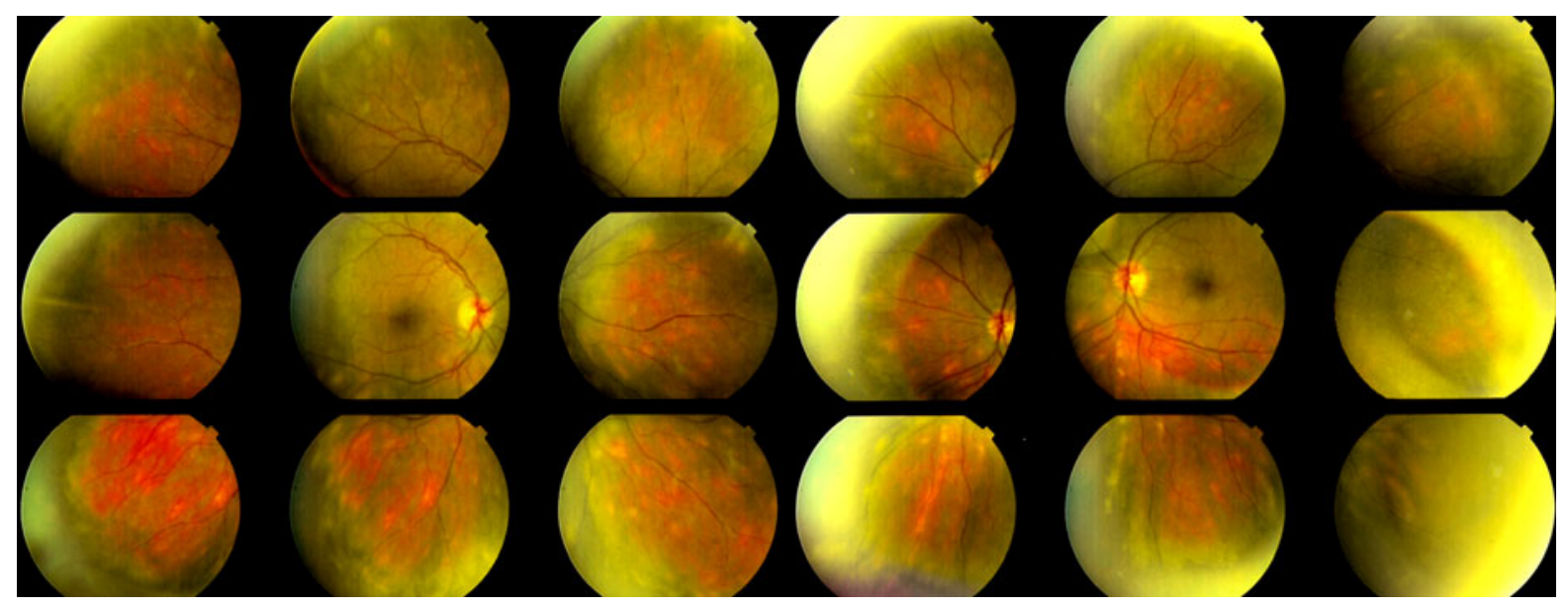

Fig. 2 Fundus photographs of patient 17 showing typical oval-shaped depigmented fundus lesions distributed in the mid-periphery 
KP could be seen in her left eye that disappeared after a subtenon's injection of triamcinolone acetonide (40 mg). Vitreous inflammation was graded as $1.5+$ in the right eye and $1+$ in the left eye. On fundus examination, FA and ICGA, all features typical of BRC could be identified as in patient 12 (Fig. 2).

Patient 18 presented at the age of 52 years, with an onset of symptoms one year before. The patient had been refusing therapy for 3 years. At the time when two KPs were observed in his left eye (Fig. 2), there was substantial anterior chamber inflammation present with flare values of $15.2 \mathrm{ph} / \mathrm{ms}$ in the right eye and $15.7 \mathrm{ph} / \mathrm{ms}$ in the left eye as well as a $2+$ vitreous infiltration. On fundus examination, FA and ICGA, all features typical for BRC could be identified as in the two previous patients (Fig. 3b). As all other inflammatory parameters had also worsened, the patient finally accepted topical and systemic IST therapy which led to the disappearance of the KPs.

\section{Discussion}

We present here, to the best of our knowledge, the first report of a considerable proportion of patients with an obvious diagnosis of BRC, not fitting into the RCBRC due to the presence of KPs. Furthermore, these KPs were of the granulomatous type, bringing 'ipso facto' BRC into the group of granulomatous uveitis entities.

A more refined and prompt clinical appraisal is necessary, since BRC is an inflammatory condition which, if not treated early, has a deleterious prognosis regarding visual function in a large proportion of cases. This will be the result of further clinical research, needing sound international elaborated and accessible diagnostic criteria for a global audience [10]. However, these criteria must be phrased with caution since exclusion criteria will diminish the already sparse cohorts of patients. The RCBRC report does not state why they chose the absence of KPs as one of the 'sine qua non' criteria for the diagnosis of BRC. The reason why granulomatous KPs are rare is because anterior segment inflammation is very scarce with low flare values not prone to produce anterior inflammatory signs such as KPs [11]. We found three patients out of 19 showing granulomatous KPs (Fig. 3a), all with the classical fundus appearance, FA signs and ICGA features of BRC (Figs. 1, 2, and 3b). Two patients each presented one single granulomatous
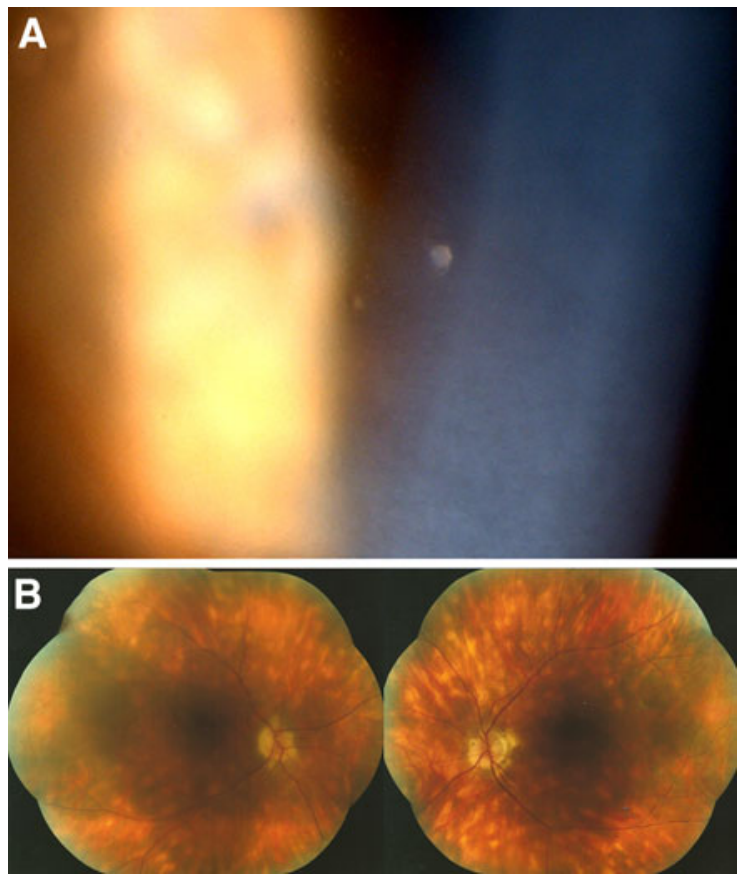

Fig. 3 a Left eye of patient 18 showing one large and one small granulomatous keratic precipitate. Considering the RCBRC, this patient, together with patients 12 and 17, would not be allowed to be included in a study, despite being HLA-B29-positive and showing all other features of BRC. b Fundus photographs of patient 18 showing typical oval-shaped depigmented fundus lesions distributed in the mid-periphery

$\mathrm{KP}$ in one eye and the third patient showed two KPs in one eye. There was a tendency in all patients to have higher anterior chamber flare levels than the other patients of the cohort.

Our three patients with KPs presented with characteristic fundus, ICGA and FA findings of BRC and were all HLA-A29-positive, excluding other possible diagnoses.

Uveitis, as proposed by Woods in 1961, is classically divided into the two main groups-non-granulomatous and granulomatous uveitis [12]. It is a helpful subdivision in the appraisal of uveitis cases, as it substantially helps to orient investigations for the establishment of diagnosis [12]. This terminology is purely based on clinical signs of inflammation in the anterior segment and in particular on the type of KPs on the endothelium. Non-granulomatous uveitis is characterized by KPs appearing as dust on the endothelium and includes among others, Behçet's uveitis and HLA-B27-related uveitis [13, 14]. All uveitis cases showing KPs that can be individualized 
should be included in the group of granulomatous uveitis [15]. The aspect of granulomatous KPs goes from micro-granulomatous KPs such as in Fuchs' uveitis to large 'mutton-fat' KPs found in ocular sarcoidosis or tuberculosis $[16,17]$. This terminology is in fact a misnomer as it uses a histological terminology to design clinical characteristics and presentations. Similarly when we talk of retinal vasculitis in a clinical setting this is also a misnomer as it uses a histological term, vasculitis, to describe clinical findings of vascular sheathing on fundoscopy or leaking on fluorescein angiography. The group of granulomatous diseases includes both histological granulomatous conditions such as ocular sarcoidosis or ocular tuberculosis and histological non-granulomatous conditions [18]. For Vogt-Koyanagi-Harada (VKH) disease non-granulomatous histological characteristics as well as granulomatous features were described depending on the evolutionary stage [19]. Unlike VKH disease, histological data for BRC are still scarce and histological findings were described as non-granulomatous in two cases reported to date [9, 12]. For the clinician it is important to understand that the clinical terms of granulomatous and non-granulomatous are not based on histological criteria but on clinical signs that go back the 1960s and allow a clinically meaningful subclassification of uveitis [12].

In conclusion, the presented study supports our motion to re-evaluate the RCBRC, since KPs can clearly be found, are granulomatous, and are more frequent than previously anticipated. Stringent application of the criteria would lead to a loss of perfectly eligible cases. Furthermore, this study provides evidence that BRC is a granulomatous disease and should be classified as such.

\section{References}

1. Ryan SJ, Maumenee AE (1980) Birdshot retinochoroidopathy. Am J Ophthalmol 89:31-45
2. Henderly DE, Genstler AJ, Smith RE, Rao NA (1987) Changing patterns of uveitis. Am J Ophthalmol 103:131-136

3. Tran VT, Auer C, Guex-Crosier Y, Pittet N, Herbort CP (1994) Epidemiology of uveitis in Switzerland. Ocul Immunol Inflamm 2:169-176

4. McCannel CA, Holland GN, Helm CJ et al (1996) Causes of uveitis in the general practice of ophthalmology. UCLA community-based uveitis study group. Am J Ophthalmol 121:35-46

5. Merrill PT, Kim J, Cox TA et al (1997) Uveitis in the southeastern United States. Curr Eye Res 16:865-874

6. Rodriguez A, Calonge M, Pedroza-Seres M et al (1996) Referral patterns of uveitis in a tertiary eye care center. Arch Ophthalmol 114:593-599

7. Gasch AT, Smith JA, Whitcup SM (1999) Birdshot retinochoroidopathy. Br J Ophthalmol 83:241-249

8. Gaudio PA, Kaye DB, Crawford JB (2002) Histopathology of birdshot retinochoroidopathy. $\mathrm{Br} \mathrm{J}$ Ophthalmol 86:1439-1441

9. Pulido JS, Canal I, Salomao D et al (2012) Histological findings of birdshot chorioretinopathy in an eye with ciliochoroidal melanoma. Eye (Lond) 26:862-865

10. Levinson RD, Brezin A, Rothova A, Accorinti M, Holland GN (2006) Research criteria for the diagnosis of birdshot chorioretinopathy: results of an international consensus conference. Am J Ophthalmol 141:185-187

11. Herbort CP, Guex-Crosier Y, de Ancos E, Pittet N (1997) Use of laser flare photometry to assess and monitor inflammation in uveitis. Ophthalmology 104:64-71 discussion 71-62

12. Woods AC (1961) Endogenous inflammation of the uveal tract. Williams and Wilkins, Baltimore

13. Rothova A, Buitenhuis HJ, Christiaans BJ et al (1983) Acute anterior uveitis (AAU) and HLA-B27. Br J Rheumatol 22:144-145

14. Tugal-Tutkun I (2009) Behcet's Uveitis. Middle East Afr J Ophthalmol 16:219-224

15. Herbort CP (2009) Appraisal, work-up and diagnosis of anterior uveitis: a practical approach. Middle East Afr J Ophthalmol 16:159-167

16. Bonfioli AA, Curi AL, Orefice F (2005) Fuchs' heterochromic cyclitis. Semin Ophthalmol 20:143-146

17. Gupta A, Bansal R, Gupta V, Sharma A, Bambery P (2010) Ocular signs predictive of tubercular uveitis. Am J Ophthalmol 149:562-570

18. Cassoux N, Charlotte F, Rao NA et al (2005) Endoretinal biopsy in establishing the diagnosis of uveitis: a clinicopathologic report of three cases. Ocul Immunol Inflamm 13:79-83

19. Rao NA (2007) Pathology of Vogt-Koyanagi-Harada disease. Int Ophthalmol 27:81-85 